\title{
Productivité des cochons d'Inde (Cavia porcellus) supplémentés au Commelina benghalensis et au Moringa oléifera.
}

Abossèdé Murielle Lucrèce Faïhun ${ }^{1}$, Coovi Guénolé Akouedegni, ${ }^{1}$ Goué Georcelin Alowanou$^{1}$, Pascal Abiodoun Olounladé, Alex Gbêliho Zoffoun ${ }^{2}$, Sylvie Mawulé Hounzangbé-Adoté ${ }^{1}$

${ }^{1}$ Laboratoire d'Ethnopharmacologie et de Santé Animale, Faculté des Sciences Agronomiques, Université d'Abomey-Calavi, 01 BP 526 Cotonou, Bénin.

2Institut National des Recherches Agricoles du Benin (INRAB), 01 B.P. 884, Cotonou, Benin.

${ }^{3}$ Laboratoire de Sciences Animales et Halieutiques (LaSAH), Unité de Recherche en Santé Globale et Environnement (URSGE), Université Nationale d'Agriculture (UNA), 01 BP : 55 Porto-Novo, Bénin.

Auteur correspondant:faibun@yahoo.fr; Téléphone:0022966551857

Mots-clés : performances de production, Cavia porcellus, supplémentation, Moringa oléfera, Commelina benghalensis.

Keywords: production performances, Cavia porcellus, supplementation, Moringa oléifera, Commelina benghalensis.

Publication date 31/08/2019, http://www.m.elewa.org/JAPS

\section{RESUME}

La présente étude a permis d'évaluer les paramètres de reproduction, de croissance et d'ingestion alimentaire des cochons d'Inde supplémentés au Commelina benghalensis ou au Moringa oleifera. Cinq rations expérimentales : ration $\mathbf{T}$ à base uniquement de son de blé, ration M20 composée du son de blé et du Moringa oléifera à $20 \%$ d'incorporation, les rations C10, C20 et C30 composées de son de blé contenant Commelina benghalensis respectivement aux taux d'incorporation de $10 \%, 20 \%$ et $30 \%$ ont été servis pendant la gestation et l'allaitement à 30 femelles primipares de 8 mois d'âge repartis en cinq lots avec trois répétitions. Le taux de fertilité a été de $100 \%$ dans tous les lots avec une taille moyenne des portées variant de 1,83 à 2,17. Le taux de mortinatalité a été élevé chez les cochonnets du lot M20 (30,77\%). Les poids de naissance des cochonnets ont varié de 74,08g à 82,07g. Le gain moyen quotidien au cours de l'allaitement a été plus élevé $\left(3,91 \mathrm{~g}^{-1}\right)$ chez les cochonnets du lot C20. La consommation de complément a été plus élevée dans le lot M20 $\left(19,30 \mathrm{~g}^{-1}\right)$. Des résultats de cette étude il ressort que Commelina benghalensis peut être recommandé dans l'alimentation des cochons d'Inde en reproduction et en période d'allaitement à un taux optimal d'inclusion de $20 \%$

\section{ABSTRACT}

The purpose of the present trial is to assess reproduction parameters, feed intake and growth of guinea pigs supplemented with Commelina benghalensis or with Moringa oleifera. Five experimental diets : diet $\mathrm{T}$ based on wheat bran, diet M20 with wheat bran and $20 \%$ of Moringa oleifera leaves powder as supplement, diets C10, C20 and C30 containing wheat bran and graded levels $(10 \%, 20 \%$ and $30 \%)$ of Commelina benghalensis over ground parts dried and mixed as supplement. They were served during the gestation and lactation period to thirty primiparous females eight months aged and divided in five batches and three repetitions. The fertility rate was $100 \%$ in all batches with the average litter size ranging between 1.83 and 2.17 . The mortinatality was high in piglets of batch M20 (30.77\%). 
The weight at birth of piglets varied from $74,08 \mathrm{~g}$ to $82,07 \mathrm{~g}$. The daily weight gain during the lactation period was high $\left(3,91 \mathrm{~g} \mathrm{j}^{-1}\right)$ in piglets of batch M20. The consumption of experimental diet was higher in batch M20 $\left(19,30 \mathrm{~g} \mathrm{j}^{-1}\right)$. At the end of this study, the results obtained allow to recommend Commelina benghalensis in guinea pigs feed during the cycle of reproduction and the lactation period. The optimal incorporation rate recommended is $20 \%$.

\section{INTRODUCTION}

Dans les pays africains, la démographie galopante surtout dans les milieux urbains, la croissance des revenus et le changement des habitudes alimentaires indiquent qu'entre 2030 et 2050 la demande des populations en produits animaux sera de deux à huit fois plus importante. Cette demande croissante en protéines d'origine animale de la population ne sera pas accompagnée d'une augmentation correspondante de la production, ce qui entraînera des incidences négatives sur la sécurité alimentaire et nutritionnelle de plusieurs ménages (Union Africaine-Bureau Inter-Africain des Ressources Animales, 2015). Des importations massives de produits d'origine animale seraient envisagées. Elles entraineront un ralentissement des économies locales par perte des recettes publiques, une difficulté d'investissement dans d'autres secteurs économiques pour lutter efficacement contre la pauvreté et l'insécurité alimentaire (CEDEAO et CSAO/OCDE 2008). En réponse à ces futurs problèmes et contraintes, la mise sur pied des élevages à cycles courts s'avère l'une des solutions durables (Organisation des Nations Unies, 2005) Au nombre de ces animaux à cycle court figurent les espèces non-conventionnelles telles que les aulacodes, les cochons d'Inde, les autruches, les porcs-épics, les escargots géants, etc. (Mensah et Ekue, 2003). Le cochon d'Inde appelé encore cobaye du nom scientifique Cavia porcellus est un

\section{MATERIELS ET METHODES}

3.1 Situation du site expérimental : L'expérimentation a été conduite entre mars et mai 2018 à la Ferme d'Application et de Recherche de la Faculté des Sciences Agronomiques (FSA) de l'Université petit rongeur originaire des régions andines de l'Amérique latine (Pérou, Colombie, Equateur et Bolivie) (Boussarie, 2003). Il présente une capacité reproductive très élevée, c'est une espèce prolifique (Lammers et al., 2009). Le nombre de petits par portée varie de 1 à 6 avec une moyenne de 3 à 4 (Bonnet, 2006). Malheureusement la productivité des cochons d'Inde à l'intérieur des élevages africains reste faible tributaire du mode d'élevage qui est du type familial et qui se pratique traditionnellement à l'intérieur des habitations (Faihun et al., 2017). L'alimentation reste encore l'un des principaux handicaps au développement de ce type d'élevage, elle est souvent déséquilibrée (faible teneur en énergie et protéines) (Miegoue et al., 2016). Selon Bindelle et Picron (2013), une amélioration de l'alimentation des cochons d'Inde peut permettre d'intensifier la production en vue de profiter de la prolificité, de la précocité et de la rusticité de ces derniers. L'une des solutions la plus envisagée et qui demeure moins coûteuse est l'utilisation des ressources fourragères locales reconnues de bonne valeur nutritive (Mweugang et al., 2016). L'objectif de la présente étude est d'évaluer les performances de production et le coût de production chez les femelles de cochons d'Inde et leur portée supplémentée au Commelina benghalensis ou au Moringa oleifera.

d'Abomey-Calavi (UAC) localisée au sud du Territoire béninois. Le climat qui y règne est de type guinéen caractérisé par deux saisons sèches et deux saisons pluvieuses. La pluviométrie moyenne annuelle est voisine de $1200 \mathrm{~mm}$. Les 
températures moyennes mensuelles varient entre 27 et $31^{\circ} \mathrm{C}$.

\subsection{Dispositif expérimental et conduite} de l'essai : Quarante-cinq (45) cochons d'Inde de race locale dont 30 femelles primipares et 15 mâles âgés d'environ 8 mois et pesant $488,58 \pm 64,07 \mathrm{~g}$ chez les femelles et $520,42 \pm 31,46 \mathrm{~g}$ chez les mâles ont été utilisés. Ils ont été répartis suivant cinq traitements et trois répétitions. Chaque lot affecté à un traitement composé de 6 femelles et 3 mâles était subdivisé en trois unités expérimentales. Chaque unité expérimentale formée de 2 femelles et un mâle constituait la famille de reproduction et était logée dans des cages en bois à fond grillagé de $600 \mathrm{dm}^{3}$. Les mâles ont été laissés en présence des femelles pendant 31 jours, ils ont été enlevés des cages après ce délai. L'alimentation des animaux durant toute la période du test (période de gestation et de lactation) était composée de Panicum maximum frais, récolté quotidiennement au stade épiaison complémenté avec les rations expérimentales telles que la ration $\mathrm{T}$ à base uniquement de son de blé, la ration M20 composée de son de blé avec de la poudre des feuilles de Moringa oléifera à un taux d'incorporation de $20 \%$ et les rations C10, C20 et C30 contenant respectivement en plus du son de blé, la poudre des parties aériennes de Commelina benghalensis à 10\%, 20\% et $30 \%$ de taux d'incorporation. Panicum maximum a été distribué ad libitum deux fois par jour (à $8 \mathrm{~h}$ et à 15h) aux cochons d'Inde. Le choix d'un taux d'incorporation de $20 \%$ de la poudre de Moringa oléifera pour la ration M20 a été retenu sur la base des travaux de Pamo et al.

\section{RESULTATS}

4.1 Composition chimique des rations : Commelina benghalensis contient la plus faible quantité de protéines brutes $(13,44 \%)$, ses énergies brutes et métabolisables les plus faibles ont été respectivement de 3675,9 Kcal $/ \mathrm{kg}$-MS et $1659,5 \mathrm{Kcal} / \mathrm{kg}-\mathrm{MS}$. Moringa oléifera par contre a présenté la plus forte teneur en
(2005) qui ont obtenu de meilleurs résultats avec ce taux d'incorporation. Chacune des cinq rations expérimentales a été servie une fois par jour (à 15h) à un lot composé de trois unités expérimentales à raison de $20 \mathrm{~g}$ de ration/jour/animal. L'identification des femelles ainsi que de leur portée a été faite par coloration de leur pelage. Les cochons d'Inde étaient pesés chaque semaine, au cours de la gestation et durant la lactation. Au cours de l'essai, des échantillons d'aliments ont été prélevés pour déterminer leurs compositions chimiques (matière sèche, matière minérale, fibre brute, matière grasse et protéines brutes) selon la méthode Association of Official Analytical Chemists (1990). Les refus alimentaires ont été pris quotidiennement. A la fin de l'étude, les données ont été soumises à une analyse de la variance (ANOVA) à un ou deux facteur(s) suivant le modèle linéaire généralisé. Ainsi les données sur les croissances pré-partum, post-partm et les paramètres de reproduction des reproductrices ont été soumises à ANOVA à un facteur (type de ration); les données sur la croissance présevrage des cochonnets ont été soumises à deux ANOVA à deux facteurs (sexe et ration, ration et type de naissance) et enfin les données sur la consommation alimentaire des reproductrices ont été également soumises à ANOVA à deux facteurs (ration et phases du cycle de reproduction). La comparaison multiple des moyennes a été effectuée par le test HSD de Tukey au seuil de signification de 5\% à l'aide du logiciel R.2.15.3.

protéines brutes $(28,14 \%)$. La ration $\mathrm{T}$ à base de son de blé a présenté la plus forte teneur en fibre $(42,8 \%)$, son énergie brute a été la plus élevée (4339 Kcal/kg-MS). La ration M20 a montré la plus forte teneur en protéines brutes $(19,18 \%)$ et le niveau d'énergie métabolisable le plus élevé (1945,8 Kcal/kg-MS) (Tableau 1). 
Tableau 1 : Composition chimique des différents aliments expérimentaux

\begin{tabular}{l|l|l|l|l|l|l|l}
\hline \multirow{2}{*}{$\begin{array}{l}\text { Aliments } \\
\text { Expérimentaux }\end{array}$} & \multicolumn{4}{|l|}{ Composition chimique (\%MS) } & $\begin{array}{l}\text { EB } \\
\text { (Kcal/kg- } \\
\text { MS) }\end{array}$ & $\begin{array}{l}\text { EM } \\
\text { (Kcal/kg- } \\
\text { MS) }\end{array}$ \\
\cline { 2 - 7 } & HR & MG & PB & MM & FT & \\
\hline Panicum maximum local & 12,34 & 2,65 & 19,74 & 10,28 & 32,9 & 4110,2 & 1813,1 \\
Moringa oleifera & 13,28 & 9,8 & 28,14 & 13,6 & 11,7 & 4202,1 & 1895,9 \\
Commelina benghalensis & 13,43 & 2,35 & 13,44 & 19,5 & 13,45 & 3675,9 & 1659,5 \\
Ration T & 8,78 & 6,3 & 18,76 & 5,89 & 42,8 & 4339 & 1897,5 \\
Ration M20 & 10,48 & 2,08 & 19,18 & 6,28 & 10,28 & 4289,2 & 1945,8 \\
Ration C10 & 10,17 & 2,25 & 17,64 & 11,41 & 11,54 & 4052,7 & 1834,8 \\
Ration C20 & 11,29 & 1,37 & 17,50 & 10,65 & 11,16 & 4087,2 & 1851,6 \\
Ration C30 & 11,08 & 2,40 & 17,08 & 9,14 & 10,63 & 4153,5 & 1883,9 \\
\hline
\end{tabular}

$\mathrm{HR}=$ humidité résiduelle $; \mathrm{MG}=$ matières grasses $; \mathrm{PB}=$ protéines brutes ; $\mathrm{MM}=$ cendres $; \mathrm{FT}=$ fibres totales ; $\mathrm{EB}=$ énergie brute ; $\mathrm{EM}=$ énergie métabolisable

\subsection{Croissance pré-partum des} reproductrices : Les reproductrices des lots $T$ et M20 ont montré les gains de poids les plus élevés au cours du premier et second mois de gestation, les gains de poids pour les deux périodes considérées sont de $142,46 \mathrm{~g}$ et $223 \mathrm{~g}$ pour le lot $\mathrm{T}$ correspondant respectivement à une augmentation de $29 \%$ et $46 \%$. Les valeurs obtenues pour le lot M20 sont de 145,10 g et de 234,81g correspondant respectivement à une augmentation de $28 \%$ et $46 \%$. Les femelles du lot C30 ont présenté le gain de poids le plus faible, leur gain total de poids au cours des deux mois de gestation s'est évalué à 185,72g soit une augmentation de 40,12\% .Il faut signaler que les différences entre les valeurs de gain de poids que ce soit au premier ou second mois ont été significatives au seuil de 5\% (Figure 1).

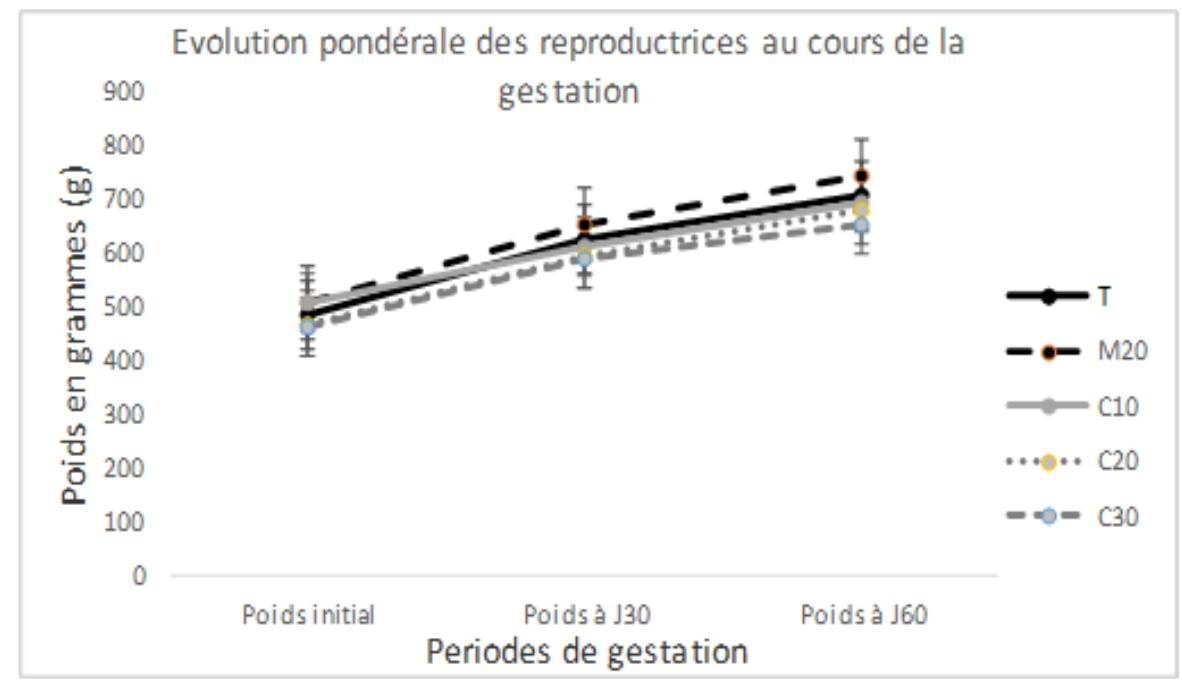

Figure 1 : Croissance pré-partum des reproductrices

$\mathrm{T}$ : lot des reproductrices ayant reçu la ration T; M20 : lot des reproductrices ayant reçu la ration M20, C10 : lot des reproductrices ayant reçu la ration C10; C20: lot des reproductrices ayant reçu la ration C20; C30: lot des reproductrices ayant reçu la ration C30.

4.3 Paramètres de reproduction des reproductrices : Le temps écoulé entre la mise au mâle et la parturition a été plus long chez les reproductrices du lot $\mathrm{C} 20$ et $\mathrm{C} 30$ (environ 81 jours); les femelles du lot $\mathrm{T}$ ont mis bas après un délai plus court (77 jours). Les femelles des lots $\mathrm{T}$ ont présenté les valeurs les plus élevées pour les paramètres tels que taux de fécondité et taux de prolificité respectivement $200 \%$ et $216,67 \%$. Les reproductrices du lot M20 ont 
présenté également une valeur élevée pour le taux de prolificité $(216,67 \%)$ mais elles ont donné le taux de fécondité le plus faible (150\%). Le taux de fertilité a été de 100\% dans tous les lots avec une taille moyenne des portées variant de 1,83 à 2,17. La différence entre les valeurs de ces différents paramètres susmentionnés n'a pas été significative d'un lot de reproductrice à un autre. Le taux de mortinatalité a été élevé chez les cochonnets du lot M20 (30,77\%). Le taux de mortalité présevrage des cochonnets enregistrée dans les lots $\mathrm{T}$ et C30 était de $16,67 \%$ et de $22,22 \%$ respectivement. Les différences ont été significatives pour ces deux paramètres d'un lot à un autre. (Tableau 2). Le type de naissance (naissance simple, double et triple) a été influencé significativement $(\mathrm{P}<0,05 \%)$ par le type de ration offert aux reproductrices. Les reproductrices ayant reçu les rations à base de Commelina benghalensis ont eu plus des portées gémellaires (un taux de $66,67 \%$ pour le lot $\mathrm{C} 10$ et un taux de $83,33 \%$ pour les lots C20 et C30). Deux portées de triplets $(33,33 \%)$ ont été enregistrées respectivement dans les lots $\mathrm{T}$ et M20. Les mortalités à la naissance des cochonnets dans les lots M20 et C10 n'ont concerné que les naissances triples. Ainsi, les taux de viabilité pour ces traitements ont été respectivement de $69,23 \%$ et $83,33 \%$. Tous les cochonnets nés vivants dans les lots C10, C20 et C30 ont été viables au sevrage par contre des cas de mortalité au sevrage ont été enregistrés dans les lots $\mathrm{T}$ (taux de mortalité avant sevrage de 9,09\%) et M20 (taux de mortalité avant sevrage de 22,22\%) (Tableau 2). 
Tableau 2: Paramètres de reproduction des reproductrices

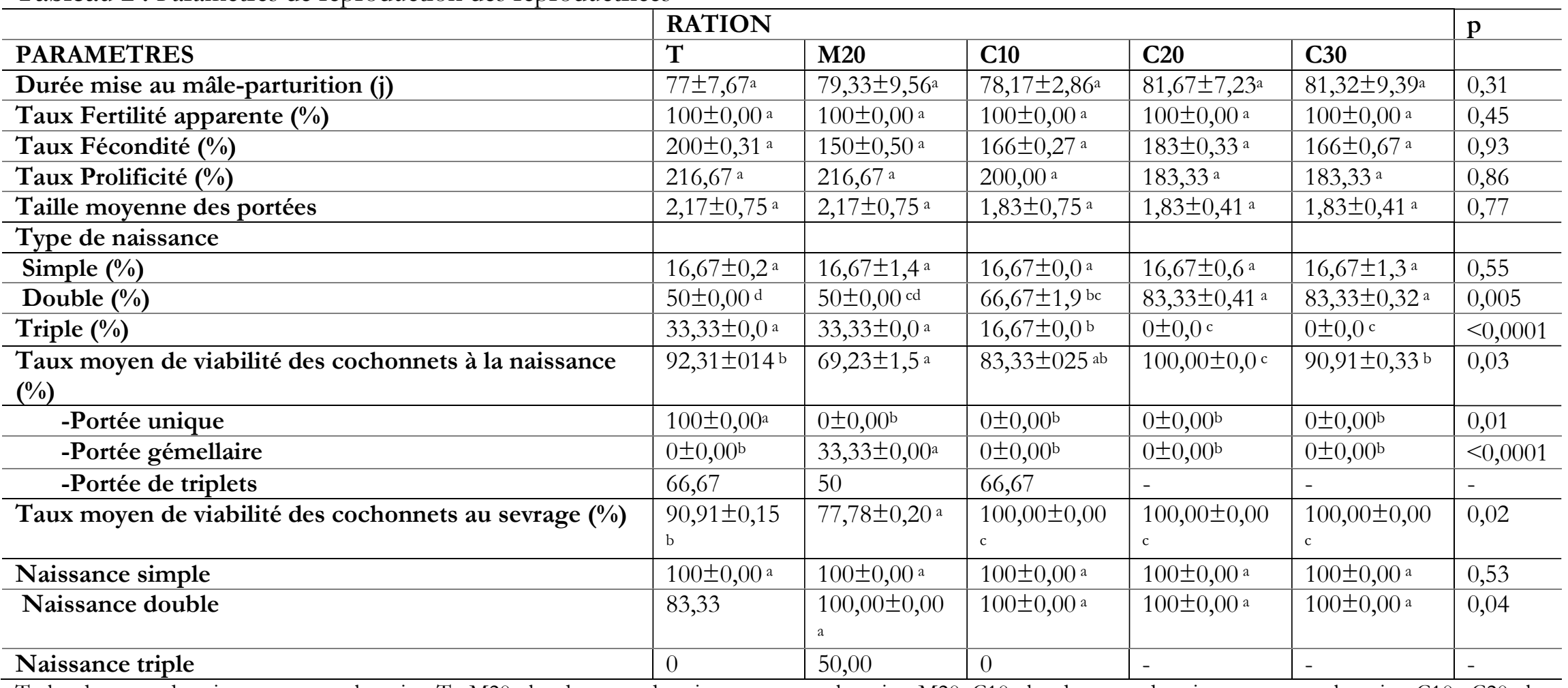

$\mathrm{T}$ : lot des reproductrices ayant reçu la ration T; M20 : lot des reproductrices ayant reçu la ration M20, C10 : lot des reproductrices ayant reçu la ration C10; C20 : lot des reproductrices ayant reçu la ration C20; C30 : lot des reproductrices ayant reçu la ration C30. (a,b) les moyennes portant les mêmes lettres sur la même ligne ne sont pas significativement différentes au seuil de 5\%; 
4.4 Croissance pré-sevrage et postpartum des cochonnets et des mères : Les cochonnets nés des reproductrices du lot T ont eu les poids les plus élevés à la naissance $(81,61 \pm 9,30 \mathrm{~g})$ et ceux nés des reproductrices du lot C30 ont eu les plus faibles poids à la naissance $(74,08 \pm 10,51 \mathrm{~g})$. Les cochonnets du lot C20 ont eu le gain de poids le plus élevé $(3,91 \mathrm{~g} / \mathrm{j})$; les valeurs de GMQ des cochonnets des autres lots sont restés comparables $(\mathrm{P}>0,05)$ (Tableau 3). Les reproductrices du lot M20 ont perdu plus de poids $(17,37 \%$ de perte de poids) alors que celles des lots T et C30 ont montré les pertes de poids les plus faibles (respectivement $14,33 \%$ et $14,43 \%$ de perte de poids) à la fin de la période de lactation (Figure 2).

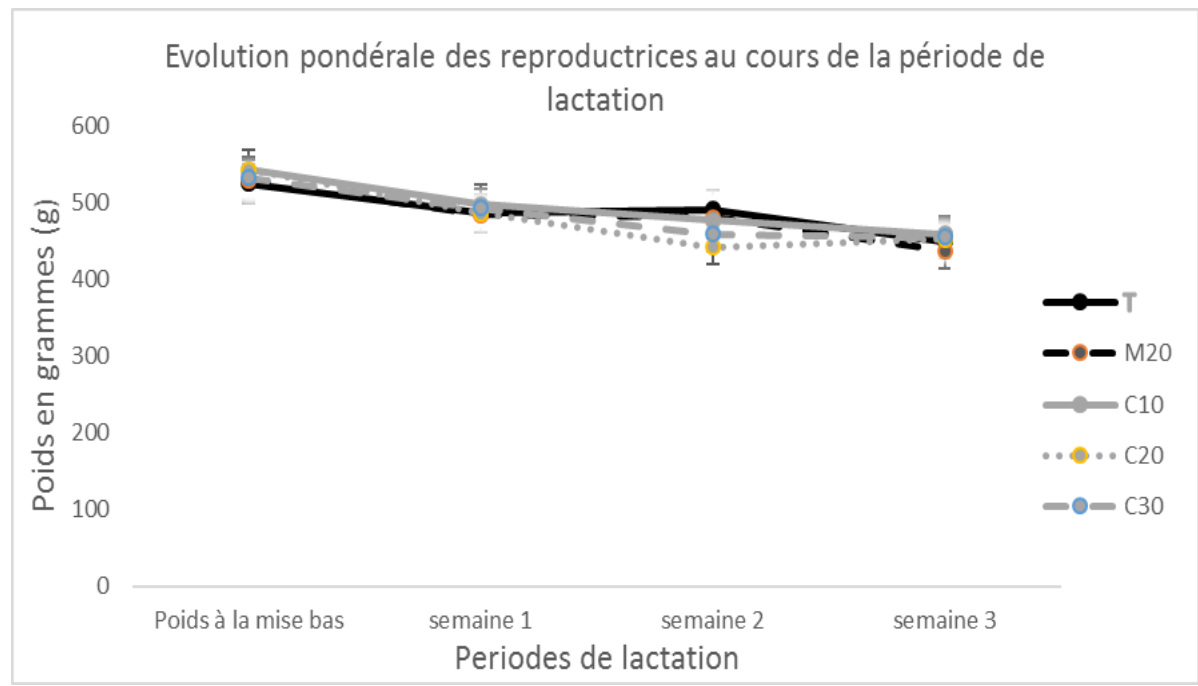

$\mathrm{T}$ : lot des reproductrices ayant reçu la ration $\mathrm{T} ; \mathrm{M} 20$ : lot des reproductrices ayant reçu la ration $\mathrm{M} 20$, C10 : lot des reproductrices ayant reçu la ration $\mathrm{C} 10$; C20 : lot des reproductrices ayant reçu la ration C20; C30: lot des reproductrices ayant reçu la ration C30.

Figure 2 : Croissance post-partum des reproductrices

En prenant en compte le sexe des cochonnets, les mâles ont généralement présenté les poids à la naissance les plus élevés à l'exception des cochonnets femelles issues des reproductrices du lot M20 qui ont eu des poids plus élevés que les mâles. Au cours de la période de lactation, les jeunes femelles ont en général vite cru que les jeunes mâles Les valeurs du GMQ ont varié de $2,87 \mathrm{~g} / \mathrm{j}$ à $3,63 \mathrm{~g} / \mathrm{j}$ chez les mâles contre $3,39 \mathrm{~g} / \mathrm{j}$ à $4,19 \mathrm{~g} / \mathrm{j}$ chez les femelles (Figure 3). Les cochonnets issus de portée unique ont été significativement $(\mathrm{p}<0,05)$ plus lourds dans tous les lots à l'exception du lot C30 où les jumeaux ont eu un poids plus lourd à la naissance $(74,40 \mathrm{~g})$. Pendant la période de lactation, les portées simples des lots $\mathrm{C} 10$ et C20 ont eu les GMQ les plus élevés $(4,88 \mathrm{~g} / \mathrm{j}$ et $4,94 \mathrm{~g} / \mathrm{j})$. Aussi les triplets des lots T, C10 et C30 ont présenté des GMQ plus élevés que les jumeaux issus de ces mêmes lots (Tableau 3 et Figure 4). 
Tableau 3 : Croissance pondérale des cochonnets au cours de la période de lactation

\begin{tabular}{|c|c|c|c|c|c|c|}
\hline \multirow[b]{2}{*}{ Paramètres } & \multicolumn{5}{|c|}{ Rations } & \multirow[t]{2}{*}{$p$} \\
\hline & $\mathrm{T}$ & M20 & $\mathrm{C} 10$ & $\mathrm{C} 20$ & C30 & \\
\hline $\begin{array}{l}\text { Pds moy } \\
\text { naissance } \\
\text { (g) }\end{array}$ & $82,07 \pm 9,30^{\text {a }}$ & $76,14 \pm 8,28 \mathrm{ab}$ & $74,36 \pm 14,15^{b}$ & $79,02 \pm 9,14 \mathrm{ab}$ & $74,08 \pm 10,51^{b}$ & 0,02 \\
\hline $\begin{array}{l}\text { Pds moy au } \\
\text { sevrage (g) }\end{array}$ & $\begin{array}{l}136,62 \pm 35,57 \\
c\end{array}$ & $151,86 \pm 7,67 \mathrm{ab}$ & $\begin{array}{l}152,33 \pm 22,62 \\
\text { ab }\end{array}$ & $161,33 \pm 32,05$ & $\begin{array}{l}143,18 \pm 19,72 \\
b\end{array}$ & 0,01 \\
\hline GMQ (g/j) & $3,25 \pm 1,36$ a & $3,25 \pm 0,82^{\text {a }}$ & $3,32 \pm 0,91^{a}$ & $3,91 \pm 1,05$ a & $3,21 \pm 0,78^{a}$ & 0,82 \\
\hline $\begin{array}{l}\text { Pds } \\
\text { naissance } \\
\partial^{\lambda}(\mathrm{g})\end{array}$ & $82,90 \pm 8,38^{\mathrm{Aa}}$ & $72,89 \pm 7,63^{\mathrm{Ad}}$ & $78.98 \pm 12.90^{\mathrm{Ab}}$ & $84.57 \pm 9.78^{\mathrm{Aa}}$ & $77,97 \pm 6,38^{\mathrm{Ac}}$ & 0,04 \\
\hline \multirow{2}{*}{$\begin{array}{l}\text { Pds } \\
\text { naissance } \\
+(\mathrm{g})\end{array}$} & $80,77 \pm 10,32^{\mathrm{Aa}}$ & $79,47 \pm 5,93 \mathrm{Bb}$ & $72.82 \pm 14.93^{\mathrm{Bc}}$ & $77.80 \pm 6.27 \mathrm{Bb}$ & $69,41 \pm 13,24^{\mathrm{Bd}}$ & 0,002 \\
\hline & $p=0,74$ & $p=0,004$ & $\mathrm{p}=0,001$ & $\mathrm{p}=0,001$ & $\mathrm{p}=0,02$ & \\
\hline $\begin{array}{l}\text { Pds } \\
\text { naissance } \\
\text { simple(g) }\end{array}$ & - & $84,77 \pm 0,00^{\mathrm{Bc}}$ & $93,18 \pm 0,00^{\mathrm{Ab}}$ & $97,15 \pm 0,00 \mathrm{Aa}$ & $\underset{\text { Ad }}{72,40 \pm 10,15}$ & 0,03 \\
\hline $\begin{array}{l}\text { Pds } \\
\text { naissance } \\
\text { double(g) }\end{array}$ & $86,86 \pm 7,29 \mathrm{Ad}$ & $\begin{array}{c}76,78,94 \pm 3,59 \\
\mathrm{Ab}\end{array}$ & $78,90 \pm 6,48^{\mathrm{Ba}}$ & $79,25 \pm 7,52^{\mathrm{b}}$ & $74,40 \pm 5,58^{c}$ & 0,02 \\
\hline \multirow{2}{*}{$\begin{array}{l}\text { Pds } \\
\text { naissance } \\
\text { triple }(g)\end{array}$} & $74,70 \pm 7,97 \mathrm{Ba}$ & $70,56 \pm 15,31 \mathrm{Ca}$ & $41,00 \pm 0,00 \mathrm{Cb}$ & - & - & $<0,001$ \\
\hline & $\mathrm{p}=0,04$ & $\mathrm{p}=0,001$ & $\mathrm{p}<0,001$ & & & \\
\hline
\end{tabular}

Pds naissance $=$ poids des cochonnets à la naissance $; \mathrm{Pds}$ au sevrage $=$ poids des cochonnets au sevrage $\mathrm{GMQ}=$ gain moyen quotidien ; $(\mathrm{a}, \mathrm{b})$ les moyennes portant les mêmes lettres sur la même ligne ne sont pas significativement différentes au seuil de $5 \%$; (A,B) les moyennes pour un paramètre donné portant les mêmes lettres sur la même colonne ne sont pas significativement différentes au seuil de $5 \%$;
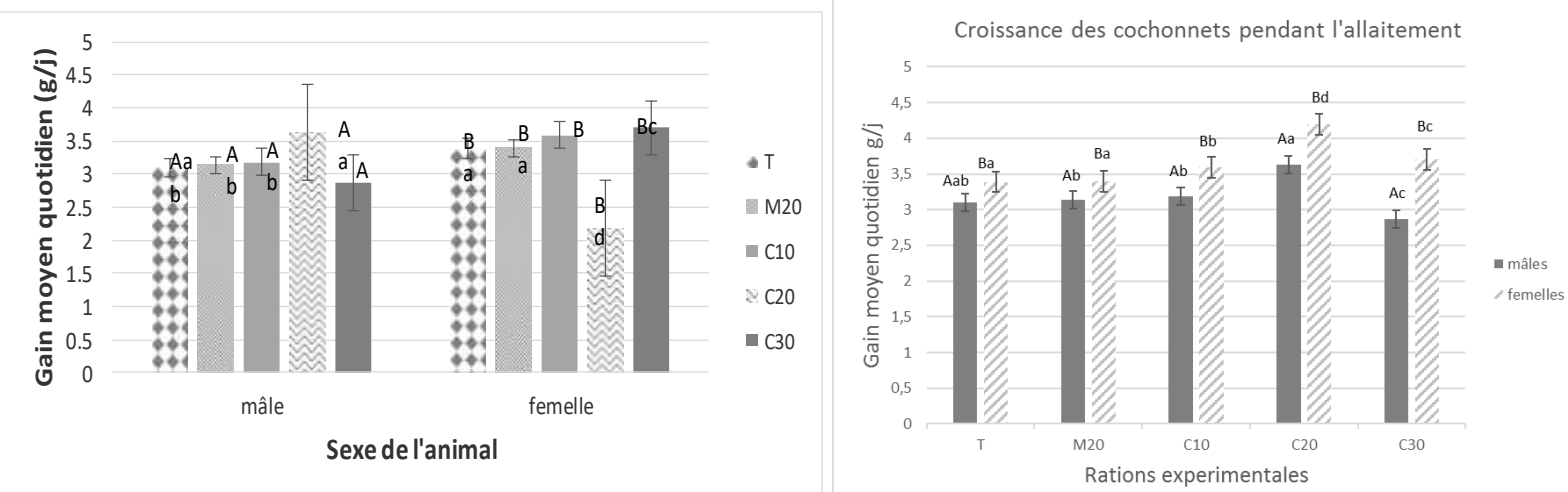

$\mathrm{T}$ : lot des reproductrices ayant reçu la ration T; M20: lot des reproductrices ayant reçu la ration M20, C10 : lot des reproductrices ayant reçu la ration C10; C20: lot des reproductrices ayant reçu la ration C20; C30: lot des reproductrices ayant reçu la ration C30. (a, b) Les moyennes portant les mêmes lettres pour un sexe donné ne sont pas significativement différentes au seuil de $5 \%$; (A, B) Les moyennes pour les deux sexes recevant la même ration portant les mêmes lettres ne sont pas significativement différentes au seuil de $5 \%$

Figure 3 : Croissance pondérale des cochonnets au cours de l'allaitement en fonction du sexe 


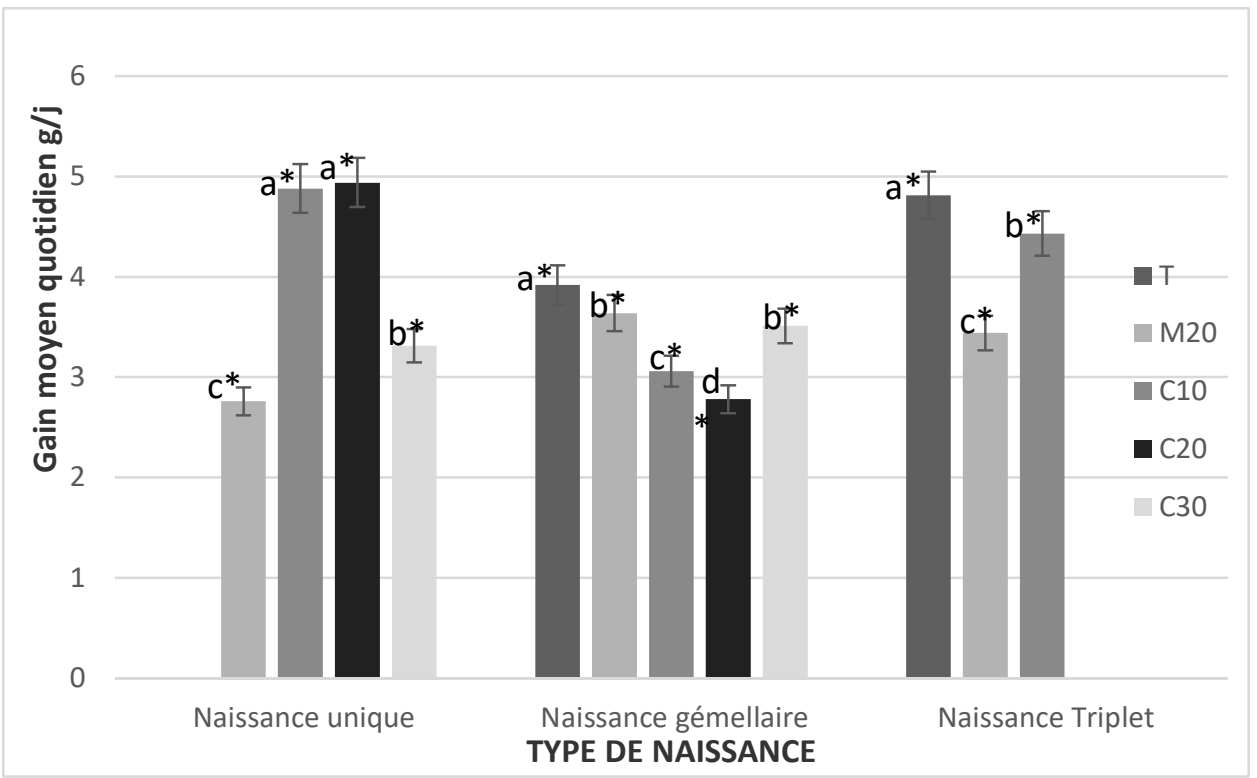

Pour cette figure, respecter les mêmes consignes que pour les figures précédentes

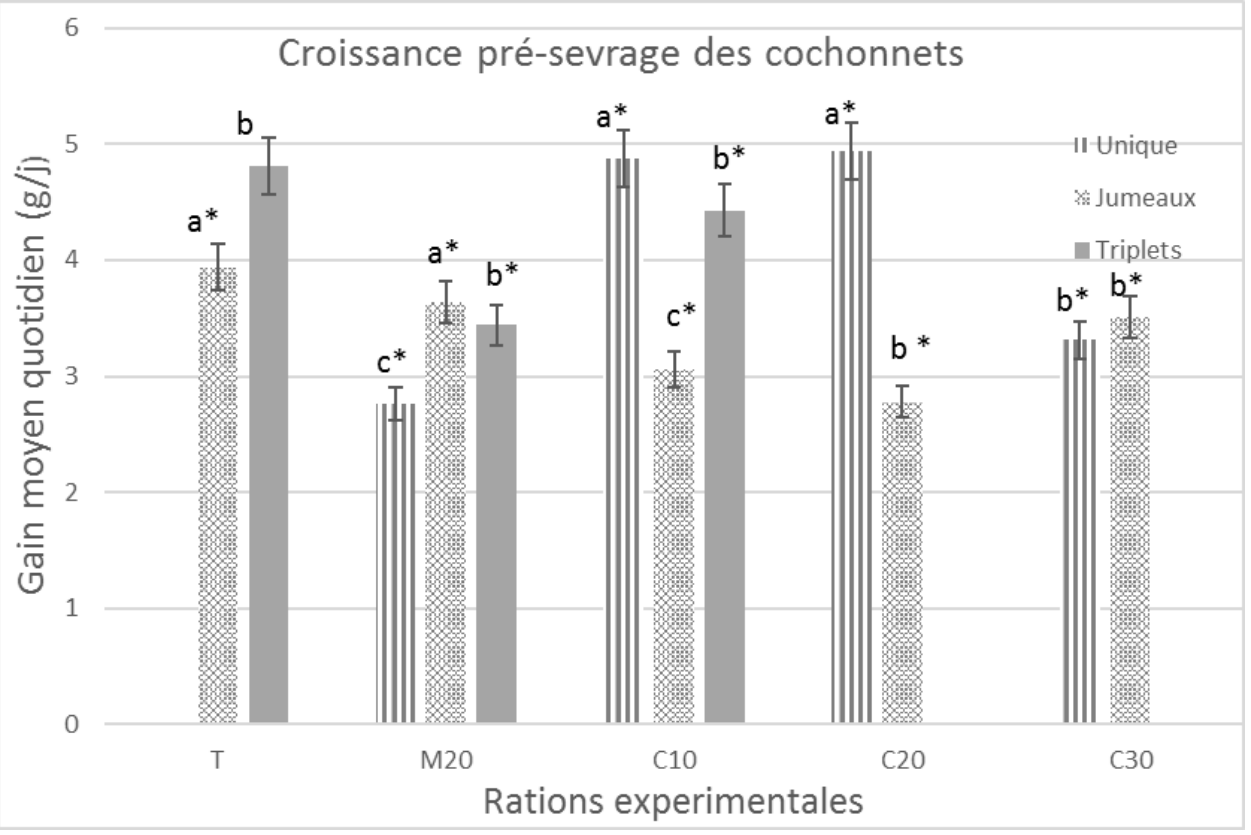

$\mathrm{T}$ : lot des reproductrices ayant reçu la ration T ; M20 : lot des reproductrices ayant reçu la ration M20, C10 : lot des reproductrices ayant reçu la ration C10; C20 : lot des reproductrices ayant reçu la ration C20 ; C30 : lot des reproductrices ayant reçu la ration C30. (a, b) Les moyennes pour les types de naissance d'un même lot portant la même lettre ne sont significativement différentes au seuil de $5 \%(*)$ les moyennes pour le même type de naissance dans les différents lots portant le même symbole sont significativement différentes au seuil de $5 \%$

Figure 4 : Croissance pondérale des cochonnets en fonction du type de naissance

4.5 Ingestion alimentaire des $(\mathrm{p}<0,05)$ par le type de ration. La reproductrices au cours de la gestation et consommation la plus élevée a été enregistrée de l'allaitement : Au cours de la période de gestation, la consommation des compléments alimentaires a été influencée significativement chez les reproductrices du lot M20 (19,30g/j); pour ce qui est des lots $\mathrm{C} 10(18,80 \mathrm{~g} / \mathrm{j})$ et $\mathrm{C} 20$ $(18,45 \mathrm{~g} / \mathrm{j})$ les consommations étaient aussi 
importantes et comparables $(\mathrm{P}>0,05)$ Les reproductrices du lot $\mathrm{T}$ ont eu la consommation alimentaire la plus faible $(13,76 \mathrm{~g} / \mathrm{j})$. A l'allaitement la consommation alimentaire des reproductrices a été plus élevée dans tous les lots mais comparativement aux valeurs obtenues au cours de la période de gestation, les différences ont été significatives seulement chez les reproductrices du lot $\mathrm{T}$ et
C30. L'ingestion alimentaire des femelles des lots $\mathrm{C} 10, \mathrm{C} 20$ et C30 a été similaire (autour de $19 \mathrm{~g})$. Les reproductrices du lot $\mathrm{T}$ ont toujours montré une ingestion alimentaire plus faible $(17,38 \mathrm{~g} / \mathrm{j})$. Au cours de cette période d'allaitement, les différences entre les consommations alimentaires d'un lot à un autre ont été significatives $(\mathrm{p}<0,05)$ (Tableau 4).

Tableau 4 : Consommation moyenne de ration expérimentale par les reproductrices au cours de la gestation et de l'allaitement

\begin{tabular}{|c|c|c|c|c|c|c|}
\hline \multicolumn{6}{|c|}{ Ingestion alimentaire de ration expérimentale $(\mathrm{g} / \mathrm{j})$} & \multirow[t]{2}{*}{$\mathrm{p}$} \\
\hline & $\operatorname{lot} \mathrm{T}$ & lot M20 & lot C10 & lot $\mathrm{C} 20$ & lot C30 & \\
\hline Gestation & $13,76 \pm 2,52^{\mathrm{cA}}$ & $19,30 \pm 1,24^{\mathrm{a} A}$ & $18,80 \pm 1,69^{a A}$ & $18,45 \pm 2,57 \mathrm{abA}$ & $17,63 \pm 3,46 \mathrm{bA}$ & $<0,001$ \\
\hline Allaitement & $17,38 \pm 3,09 \mathrm{cB}$ & $18,75 \pm 2,60^{\mathrm{bA}}$ & $19,45 \pm 1,58^{\mathrm{aA}}$ & $19,48 \pm 1,99 \mathrm{aA}$ & $19,75 \pm 0,83^{\mathrm{aB}}$ & $<0,001$ \\
\hline & $\mathrm{p}<0,001$ & $p=0,97$ & $\mathrm{p}=0,88$ & $\mathrm{p}=0,55$ & $p=0,02$ & \\
\hline
\end{tabular}

$\mathrm{T}$ : lot des reproductrices ayant reçu la ration T; M20 : lot des reproductrices ayant reçu la ration M20, C10 : lot des reproductrices ayant reçu la ration C10; C20 : lot des reproductrices ayant reçu la ration C20 ; C30: lot des reproductrices ayant reçu la ration $\mathrm{C} 30 .(\mathrm{a}, \mathrm{b})$ Les moyennes portant les mêmes lettres sur la même ligne ne sont pas significativement différentes au seuil de 5\%; (A, B) Les moyennes portant les mêtmes lettres sur une même colonne ne sont pas significativement différentes au seuil de $5 \%$.

T: M20: C10: C20: C30 :

4.6 Coût de production de poids vif à la mise-bas et au sevrage : L'analyse de la relation coût relatif des aliments expérimentaux ingérés par femelle au cours de la période de gestation, de lactation et la production de poids vif a montré que la ration $\mathrm{T}$ a induit un gain de poids meilleur à la mise bas pour un coût plus faible. Ainsi la production de $100 \mathrm{~g}$ de poids vif à la mise bas avec cette ration a coûté 133,79 FCFA. La ration C20 a permis la production de poids vif au sevrage à coût plus réduit soit 65,59 FCFA pour la production d'un croit de 100g. La ration C30 a induit un croit de poids vif à un coût plus élevé que ce soit à la mise bas (321 F CFA/100g de poids vif produit) ou au sevrage, (104 F FCA $/ 100 \mathrm{~g}$ de poids vif produit) (Tableau 5).

Tableau 5 : Coûts (en F CFA) des aliments expérimentaux distribués pour la production du poids vif à la parturition et au sevrage

\begin{tabular}{|c|c|c|c|c|c|}
\hline \multirow[b]{2}{*}{ Paramètres } & \multicolumn{5}{|c|}{ Traitements } \\
\hline & Lot $T$ & Lot M20 & Lot C10 & Lot C20 & Lot C30 \\
\hline $\begin{array}{l}\text { Production de poids vif à la parturition/femelle } \\
\text { (g) }\end{array}$ & 150,46 & 114,21 & 123,93 & 144,87 & 123,46 \\
\hline $\begin{array}{l}\text { Coût total du complément alimentaire } \\
\text { consommé au cours de la gestation/femelle } \\
\text { (FCFA) }\end{array}$ & 201,31 & 355,21 & 317,15 & 364,65 & 384,22 \\
\hline Production de poids vif au sevrage/femelle (g) & 96,31 & 88,34 & 115,23 & 150,90 & 106,35 \\
\hline $\begin{array}{l}\text { Coût total de l'alimentation consommée au cours } \\
\text { de l'allaitement/femelle (FCFA) }\end{array}$ & 69,35 & 91,35 & 88,23 & 98,99 & 111,15 \\
\hline $\begin{array}{l}\text { Coût de production de } 100 \mathrm{~g} \text { de poids vif à la } \\
\text { gestation/femelle (FCFA) }\end{array}$ & 133,79 & 311,01 & 255,91 & 251,70 & 311,21 \\
\hline $\begin{array}{l}\text { Coût de production de } 100 \mathrm{~g} \text { de poids vif au } \\
\text { sevrage/femelle (FCFA) }\end{array}$ & 72,00 & 103,41 & 76,56 & 65,59 & 104,51 \\
\hline
\end{tabular}

Prix du kilogramme de la ration T=190 FCFA, 216 FCFA pour la ration C10), 242 FCFA pour la ration C20, 268 FCFA pour la ration $\mathrm{C} 30$ et $232 \mathrm{FCFA}$ pour la ration M20 


\section{DISCUSSION}

5.1 Composition chimique des rations : L'analyse de la composition chimique des différentes espèces fourragères utilisées dans l'alimentation des cochons d'Inde lors de cette étude a révélé que Commelina benghalensis a présenté les plus faibles teneurs en protéines brutes et matières grasses qui ont été de $13,44 \%$ et $2,45 \%$ respectivement. Cette valeur de teneur en protéines est différente de celle rapportée en Inde par Vishwakarma et Dubey (2011) qui est de 8,5\% s. Lanyasunya et al. (2008) au Kenya ont rapporté une teneur en protéines brutes de Commelina benghalensis variant de $9,25 \%$ à $17,59 \%$ et une teneur en matières grasses variant de $1,14 \%$ à $2,21 \%$ suivant son stade physiologique. La teneur en protéines brutes de 28,14\% des feuilles de Moringa oléifera obtenue est comparable à celle trouvée par Pamo et al. (2005) (26,37\%) au Cameroun. Les valeurs de l'énergie métabolisable des cinq rations expérimentales (T, M20, C10, C20, C30) sont comprises entre $1700-2800 \mathrm{kcal} / \mathrm{kgMS}$ intervalle recommandé par Clarke et al. (1977) pour une croissance normale des cochons d'Inde.

5.2 Performances de reproduction: Les reproductrices du lot M20 ont eu le GMQ le plus élevé $(3,91 \mathrm{~g} / \mathrm{j})$ au cours de la période de gestation. Cette valeur est inférieure à celles obtenues par Mweugang et al. (2016) (4,99g/j) chez les cochons d'Inde complémentés à la farine de feuilles de manioc et par Kouakou et al. (2012) $(6,10 \mathrm{~g} / \mathrm{j})$ chez les cochons d'Inde complémentés aux granulés pour lapin durant toute la période de gestation. Cette valeur est cependant supérieure à celle rapportée par Pamo et al. (2005) $(1,63 \mathrm{~g} / \mathrm{j})$ chez des cochons d'Inde également complémentés au Moringa oléifera. Cette grande différence observée entre les valeurs serait liée à la manière dont les rations ont été formulées. Ces auteurs en effet ont réalisé une addition du Moringa oléifera au taux de $20 \%$ dans du son de blé alors que dans le présent essai la poudre des feuilles sèches de Moringa oléifera a été incorporé par substitution. Les cochons d'Inde ayant reçu Commelina benghalensis ont montré les plus faibles gains de poids au cours de la période de gestation. La plus faible croissance a été observée chez les reproductrices du lot C30 ce qui pousse à formuler l'hypothèse selon laquelle un fort taux d'incorporation de Commelina benghalensis empêcherait la libération et la disponibilité de nutriments aux reproductrices. Les reproductrices de cet essai sont à leur deuxième cycle de reproduction. Le taux de fertilité a été de 100\% dans tous les lots. Les cochons d'Inde supplémentés aux feuilles de manioc ont également donné un taux de fertilité de 100\% (Mweugang et al., 2016). Les cochons d'Inde supplémentés par contre avec Arachis glabrata et Desmodium intortum ont donné des taux de fertilité respectivement de $93,33 \%$ et 73,33\% (Tchoumboué et al., 2001). Les valeurs du taux de fécondité ont varié de $116,67 \%$ à $200 \%$. Les taux de prolificité ont varié de $183,33 \%$ à $216,67 \%$. Fotso et al. (1995) ont également obtenu des taux de fécondité passant de 153 à 196\% de la 1 ère à la $2^{\text {ème }}$ mise bas. La taille moyenne de portée obtenue à la fin de cet essai a varié de 1,83 à 2,17 ; les lots T et M20 ayant donné les tailles de portées les plus élevées. Ces valeurs sont meilleures à celles rapportées par Kouakou et al. (2012) $(1,2$ à 1,8) ; par Azine et al. (2016) (1,12 à 1,33). Les premiers auteurs avaient nourri les reproductrices aux granulés de lapins et les auteurs suivants avaient nourris les animaux avec Galinsoga sp., Bidens pilosa, Ageratum conysö̈dens. Ces différences observées seraient sûrement dues à la présence de composés antinutritionnels dans les plantes. Selon Francis et al. (2002) Bidens pilosa serait riche en saponine, et autres extraits inhibiteurs de la synthèse des hormones sexuelles, empêchant l'implantation de l'œuf et provoquant des avortements. Les faibles performances induites par les granulés pour lapin de bonne densité énergétique (2435,2 Kcal/kgMS) seraient peut-être dues aux facteurs intrinsèques des reproductrices. Les femelles issues de portées multiples montrent un retard dans l'atteinte de la maturité sexuelle et une réduction de la fertilité comparativement à celles issues des portées de petites tailles (Mendl, 1988). Le taux de mortinatalité a été élevé au sein des cochonnets nés des femelles du lot M20. Cette situation serait la conséquence de leur surpoids à la fin de la période de gestation se traduisant par des difficultés lors de la délivrance des petits. L'obésité affecte en effet négativement la reproduction chez les cobayes (Michel et Bonnet, 2012). Les morts-nés étaient complètement développés et parfois encore couverts de leur sac amniotique. Ceci indique que les mères n'ont probablement pas rompu les sacs amniotiques et les petits ont été asphyxiés au cours de la délivrance (Tejml et al., 2015). A la naissance le poids des cochonnets a varié de $74,08 \mathrm{~g}$ à $82,07 \mathrm{~g}$, les plus faibles étant enregistrés chez ceux du lot C30. Ceci vient conforter l'hypothèse qu'un un fort taux 
d'incorporation de Commelina benghalensis empêcherait la libération et la disponibilité de nutriments aux reproductrices. De façon générale, les mâles ont présenté les poids les plus élevés à la naissance ce qui abonde dans le sens des résultats de Nemeth et al. (2017). Le taux de mortalité avant sevrage chez les cochonnets issus de portée triple a été plus élevé. Ce résultat pourrait s'expliquer par le faible poids de naissance de ces derniers. En effet les cochonnets ayant un poids de naissance inférieur à $60 \mathrm{~g}$ ont de faible chance de survie et meurt généralement en cours de lactation (Harkness et al., 2002). Le faible poids des portées multiples serait dû à la répartition des nutriments de la mère au sein des différents fotus présents dans l'utérus (Wehmer et Jen, 1978). Les besoins pendant la lactation sont souvent difficiles à couvrir, et la femelle doit fournir en plus beaucoup d'efforts pour couvrir la demande des petits (Laurien-Kehnen et Trillmich, 2003). Elle doit donc, pour assurer la lactation, mobiliser ses réserves corporelles, ce qui expliquerait la perte de poids observée chez les femelles allaitantes. Kouakou et al. (2012) ont plutôt rapporté une augmentation du poids des femelles allaitantes nourries à base du Panicum maximum et supplémentées avec du granulé pour lapin de la mise-bas au sevrage. Aucune mortalité pré-sevrage des cochonnets soumis aux rations contenant Commelina benghalensis n'a été enregistré, ils ont sûrement reçu une qualité et une quantité de lait meilleure ce qui a favorisé leur survie. Commelina benghalensis selon Mahadkar et al. (2012) constitue une bonne source de calcium 1431,6mg/100g MS.

\subsection{Consommation alimentaire et coût de} production : Au cours de la période de gestation la consommation des rations expérimentales a varié de 13,76g à 17,63g. Les plus fortes consommations ont été enregistrées chez les reproductrices soumises aux rations contenant Moringa oleifera ce qui a sans doute induit des gains de poids élevés chez ces dernières. Aussi cette observation pourrait faire

\section{CONCLUSION}

L'incorporation de Commelina benghalensis dans l'alimentation des reproductrices a amélioré leurs paramètres de reproduction. Les cochonnets soumis aux rations contenant cette plante ont montré un taux de viabilité de $100 \%$ au sevrage. La ration la plus efficace ayant induit un gain de poids vif au sevrage à coût réduit a été la ration C20 contenant du Commelina benghalensis au taux d'incorporation de $20 \%$ dans du son de blé. La ration M20 contenant penser que cette ration avait meilleur goût ce qui a favorisé sa surconsommation. Kouakou et Brou (2016) ont enregistré une consommation de compléments alimentaires des cochons d'Inde variant de $20 \mathrm{~g}$ à $22 \mathrm{~g}$ au cours de la période de gestation. Les femelles dans cet essai ont été complémentées avec Euphorbia heterophylla associé au maïs-grain et à la farine de poisson et aux granulés pour lapin. La différence observée par rapport au niveau de consommation alimentaire des cochons d'Inde de cet essai comparativement au nôtre serait peut-être liée au mode de présentation des compléments. Les aliments étaient servis séparément, ils ne formaient pas un mélange homogène, les animaux ont donc plus consommé les aliments de leur choix de leur choix ce qui a augmenté la valeur cumulative de l'ingestion des compléments. Au cours de la période de lactation la consommation alimentaire a baissé chez les reproductrices du lot T et du Lot M20. Elle a plutôt augmenté chez les reproductrices ayant reçu Commelina benghalensis. Cette observation pourrait s'expliquer par le fait que les reproductrices des lots T et M20 avaient moins de petits à allaiter à cause des cas de mortalités de ces derniers plus enregistrés. Ainsi leurs besoins énergétiques sont moins importants ce qui se traduit par une baisse du niveau de consommation alimentaire. Le complément alimentaire le plus efficace pour la production de poids vif à la fin du sevrage a été la ration C20. Le coût de production de $100 \mathrm{~g}$ de poids vif par chaque reproductrice de ce lot s'est évalué à 65,59 FCFA. Ce coût de production se rapproche de celui obtenu par Kouakou et Brou (2016) (63,1 FCFA) pour des femelles nourris au Panicum maximum et complémentées avec Euphorbia beterophylla associé au maïs-grain. Il est également comparable au coût de production (62,5 FCFA) de $100 \mathrm{~g}$ de poids vif des femelles recevant du granulé pour lapin pendant la période de lactation (Kouakou et al., 2002).

du Moringa oléifera à 20\% de taux d'incorporation a entrainé des gains de poids élevés chez les reproductrices qui ont connu des difficultés lors de la délivrance des petits à la mise-bas se traduisant par des taux de mortinatalité élevés. Commelina benghalensis peut donc servir à alimenter les cochons d'Inde au cours de leur cycle de reproduction. Il serait peut-être plus efficient d'incorporer Commelina benghalensis à un taux de $20 \%$. Toutefois il serait utile 
d'étudier l'effet de Commelina benghalensis à l'état frais ou sous forme d'ensilage sur les performances de

\section{REFERENCES BIBLIOGRAPHIQUES}

Association of Official Analytical Chemists: 1990. Official methods of analysis. 15th ed. AOAC, Arlington, VA.

Azine P C, Niba A T, Meutchieye F and Teguia A: 2016. Performances de reproduction des cobayes (Cavai porcellus 1.) supplémentés aux astéracées ou à l'aliment composé enrichi en protéines. Bulletin of Animal Health and Production in Africa 64: 263-272.

Bindelle J and Picron P: 2012. Le Cobaye, un Petit Herbivore Facile à Nourrir dans des Petites Parcelles. Dossier spécial rongeur. Troupeaux et Cultures des Tropiques 1:8pp.

Bonnet $\mathrm{O}: 2006$. Élaboration d'un protocole de visite d'élevage des rongeurs et lagomorphes de compagnie. Thèse de Médécine Vétérinaire, Lyon. p 32-34, 43, 62-77.

Boussarie D: 2003. Consultation des petits mammifères de compagnie. Edition du point vétérinaire. 210p.

Clarke H E, Coates M E, Eva J K, Ford D J, Milner C K, O'donoghue P N, Scott P and Ward R $\mathrm{J}:$ 1977. Dietary standards for laboratory animals: report of the Laboratory Animals Centre Diets Advisory Committee. Laboratory Animals 1: 1.28.

Faihun A M L, Akouedegni C G, Olounlade P A and Hounzangbe-Adote M S: 2017. Typologie des élevages de cobayes (Cavia porcellus) au Bénin. International Journal of Biological and Chemical Sciences 11(2): 556570.

Fotso J M, Ngou Ngoupayou J D and Kouomenioc $\mathrm{J}:$ 1995. Performances expérimentales des cobayes élevés pour la viande au Cameroun. Cabiers Agriculture 4: 65-69.

Francis G, Kerem Z, Harinder M and Klaus B: 2002. The biological action of sap nine in animal system. A review. British Journal of Nutrition 88: 587-605.

Harkness J E, Murray K A and Wagner J E: 2002. Biology and diseases of guinea pigs. In: Fox JG, Anderson LC, Lowe FM, Quimby FW, edition Laboratory animal medicine. San Diego (CA): Academic Press. p. 203-246.

Kouakou N'G D V and Brou Y B S: 2016. Réduction des charges alimentaires des reproduction des cochons d'Inde.

cobayes (Cavia porcellus L.) par un régime fourrager supplémenté par la farine de poisson et/ou du maïs-grain durant le cycle de reproduction. International Journal of Biological and Chemical Science 10(3): 11991209

Kouakou D V, Thys E, Danho M, Nogbou Assidjo E, and Grongnet J F: 2012. Effet de Panicum maximum sur la productivité des femelles primipares durant le cycle de reproduction chez le cobaye (Cavia porcellus L.). Tropicultura 30 (1): 24-36.

Lammers P J, Carlson S L, Zdorkowski G A and Honeyman M S: 2009. Reducing food insecurity in developing countries through meat production: the potential of the guinea pig (Cavia porcellus). Renewable Agricultural Food Systems 24 (2): 155-162.

Lanyasunya T P, Hongrong Wang ST, Kariuki E A, Mukisira S A, Abdulrazak N K, Kibitok J O and Ondiek: 2008. The potential of Commelina benghalensis as a forage for ruminants. Animal Feed Science and Technology 144: 185-195.

Laurien-Kehnen, C. and Trillmich, F. 2003. Lactation performance of guinea pig (Cavia porcellus) does not pup demands. Behav. Ecol. Sociobiol. 53: 145-152.

Mahadkar S, Valvi S and Rathod V: 2012. Nutritional assessment of some selected wild edible plants as a good source of mineral. Asian Journal of Plant Science and Resesearch 2 (4): 468-47.

Mendl M: 1988. The effects of litter size variation on mother-offspring relationships and behavioural and physical development in several mammalian species (principally rodents). Journal of Zoology Society of London 215: 15-34.

Mensah G A and Ekue M R M: 2003. L'essentiel en aulacodiculture.

ReRE/KIT/IUCN/C.B.D.D. - République du Bénin/Royaume des Pays-Bas. 160 p.

Michel C L and Bonnet X: 2012. Influence of body condition on reproductive output in the guinea pig. Journal of Experimental Zoology 317: 24-31. 
Miegoue E, Tendonkeng F, Lemoufouet J, Noumbissi M N B, Mweugang N, Zougou G T, Boukila B, Nkouadjio M F and Pamo E T: 2016. Croissance présevrage des cobayes nourris au Panicum maximum supplémenté avec une ration contenante Arachis glabrata, Calliandra calothyrsus ou Desmodium intortum. International Journal of Biological and Chemical Science 10(1):313-325.

Mweugang N, Tendonkeng F, Miegoue E, Matumuini F E N, Zougou G T, Fonteh F A, Boukila B and Pamo, E. T : 2016. Effets de l'inclusion de feuilles de manioc (Manihot esculenta Crantz) dans la ration sur les performances de reproduction du cobaye (Cavia porcellus L.) local camerounais. International Journal of Biological and Chemical Science 10 (1): 269-280.

Nemeth M, Millesi E, Siutz C, Wagner K.H, Quint $\mathrm{R}$ and Wallner B: 2017. Reproductive performance and gestational effort in relation to dietary fatty acids in guinea pigs, Journal of Animal Science and Biotechnology 8:28.

Organisation des Nations Unies (ONU) : 2005. 'Soutien au mécanisme de survie par la fourniture urgente d'intrants agricoles aux familles vulnérables dans une situation post-conflits', in ONU and RDC, ed, Projets 2005 : Procédure d'appel global, Sécurité alimentaire, Kinshasa, 21pp.

Pamo T E, Niba A T, Fonteh F A, Tedonkeng F, Kana J R, Boukila B and Tsachoung J: 2005. Effet de la supplémentation au Moringa oleifera ou aux blocs multinutritionnels sur l'évolution du poids post partum et la croissance pré-sevrage des cobayes (Cavia porcellus L.). Livestock Resources. Rural Development 17 (4): 9p.

Tchoumboue J, Niba AT and Kenfack A: 2001. Comparative studies on the influence of supplementation with two legumes (Arachis glabrata and Desmoduim intortum) on the reproductive and growth perfomance of Guinea pigs (Cavia porcellus L.). Bulletin of Animal Health and Production in Africa 49: 7983.

Tejml P, Šoch M, Broucek J, Jirotkova D, Smutný L and Zabranský L : 2015. Factors influencing behaviour of guinea pig females during the birth. Advances in Environmental Science and Energy Planning 81-4.
Union Africaine- Bureau Inter-Africain des Ressources Animales (UA-BIRA): 2015. The Livestock Development Strategy for Africa 2015-2035. Nairobi, Kenya, 158p.

Vishwakarma K L and Dubey V: 2011. Nutritional analysis of indigenous wild edible herbs used in eastern Chhattisgarh, India Kanchan. Emirates Journal of Food and Agriculture 23 (6): 554-560.

Wehmer F and Jen K C: 1978. The effects of litter size during gestation and lactation on rat development prior to weaning. Developmental Psychobiology 11: 353-360. 